\title{
Controlled Epidemic Routing for Multicasting in Delay Tolerant Networks
}

\author{
Muhammad Abdulla and Robert Simon \\ Department of Computer Science \\ George Mason University \\ Fairfax, VA 22030 \\ Email: \{mabdulla, simon\}@cs.gmu.edu
}

\begin{abstract}
Delay tolerant networks (DTNs) are a class of networks that experience frequent and long-duration partitions due to sparse distribution of nodes. DTN multicasting is a desirable feature for applications where some form of group communication is needed. The topological impairments experienced within a DTN pose unique challenges for designing effective DTN multicasting protocols. In this paper, we examine multicasting in DTNs using controlled flooding schemes. Specifically, we analyze basic multicast routing schemes for fundamental performance metrics such as message delivery ratio, message delay, and buffer occupancy. Further, we study the effects of different controlled Epidemic routing schemes using TTL and message expiration times. We provide analytical results for performance metrics and perform extensive evaluations of our proposed methods. Our experiments show that our analytical results are accurate and that with careful protocol parameter selection it is possible to achieve high delivery rates for various system scenarios.
\end{abstract}

\section{INTRODUCTION}

Delay tolerant networks (DTNs) are a class of emerging networks that experience frequent and long duration partitions [5]. In such networks, an end-to-end path between the source and the destination may never exist. With the increased use of wireless mobile devices, many of the new network applications fall into this category of such networks, such as wildlife tracking, military networks, and disaster recovery and emergency response, etc.

In this paper we present CERM: Controlled Epidemic Routing for Multicasting, a collection of schemes to achieve efficient message delivery for multicasting through the use of controlled Epidemic routing in DTNs. Multicasting is the transmission of packets to a group of hosts identified by a single destination address (group id) and Epidemic Routing is flooding in the context of DTNs [17]. Multicasting may be desirable in many potential DTN applications where a close collaboration of participating members is necessary. For example, sensors deployed in a military field for intrusion detection may need to communicate with other nodes for a more complete information regarding the intruding object. In an emergency response scenario, rescue workers want to disseminate information regarding local condition and hazard level. While such group communication requirements can be fulfilled with separate unicast operations, power and storage restrictions as well as delivery requirements necessitate efficient group communication support in DTNs. For this purpose, we provide flexible routing schemes in CERM and analytical results for basic routing schemes to gain an understanding in multicast schemes and to achieve desired tradoffs between resource usage and message delivery performance.

Although multicasting, or routing in general, has been studied extensively for the Internet and mobile ad hoc networks (MANETs), routing in DTNs remains to be challenging problem due to long delays and frequent partitions. Since there may not exist an end-to-end path in DTNs, both proactive and reactive routing schemes fail to work. Proactive routing schemes, where nodes try to keep up to date routing information for other nodes, may fail to converge while producing a lot of periodic update packets. In reactive routing schemes, where routing information is obtained on demand, nodes mail fail to find a path to the destination. However, this does not mean that the packets cannot be delivered to the destination. Due to node mobility, different links come up and down over time, enabling nodes to achieve eventual delivery through store-carry-and-forward approach, which uses buffers to hold the message until the next link comes up in the end-to-end path. Epidemic routing is one of such schemes where nodes exchange messages that they don't have in common upon node contact to perform flooding in the context of DTNs. The purpose of CERM is to achieve high message delivery ratios and low delays while trying to decrease buffer occupancy and the number of message transfers.

The semantics of multicasting in DTNs should also be considered besides the routing challenges mentioned above. In traditional networks, data transfer delays are generally very short and group membership changes during data transfers are rare and can be ignored. However, because of frequent partitions and consequent long delays in DTNs, group membership changes require unambiguous semantic models for implementation and analysis of multicasting under such conditions [21].

A number of routing schemes assume prior knowledge of node mobility and connectivity, or oracles, to perform message transfers [8][21]. In some other cases, additional measures and network resources are used to handle disconnections among nodes. For example, a special node, or a ferry can move on a specific trajectory to help nodes on the network transfer data [20].

We examine efficient multicast routing schemes for DTNs. 
Based on group-based delivery and Epidemic routing schemes we extend current routing schemes to CERM. In CERM we use TTL, message expiration times, probabilistic routing, and other inter-group routing policies to achieve desired resourceperformance tradeoffs. We do not assume any prior knowledge of node connectivity. We focus on the performance analysis and evaluation of different controlled Epidemic multicast routing schemes. Further, we give analytical results for message delivery ratios, message delay, and buffer occupancy for basic schemes by modeling the system through the use of phase-type $(\mathrm{PH})$ distributions. Our results show that our analytical resutls are accurate and that with careful protocol parameter selection it is possible to achieve high delivery ratios for various system scenarios.

The rest of the paper is structured as follows. Section II goes over related work. Section III describes our proposed approaches for multicasting in DTNs. Section IV presents performance analysis. Section V describes performance results of the proposed approaches. Finally, Section VI concludes the paper.

\section{RELATED WORK}

Delay tolerant network concepts are discussed in $[3,5,8]$. In this category of networks, frequent partitions and long delays are common. Due to these characteristics, well-known routing schemes fail to work properly under such conditions. The basic routing paradigm for effective routing in DTNs is to use the store-carry-and-forward approach, where intermediate nodes keep the messages until new links come up in the path to the destination.

Besides the store-and-forward approach, works have been done to introduce additional network resources to improve routing performance. In [12] a number of mobiles nodes perform random walks to collect packets, buffer them, and deliver them to wired access points. On the other hand, [20] introduces non-randomness to node mobility by using message ferries that travel on a trajectory to provide communication services. Either the message ferries choose a trajectory to contact nodes, or the nodes can move near to pre-defined trajectory at a certain time to exchange packets.

Jain et al. [8] formulated DTN routing by means of directed multi-graph, where more than one edge may exist between a pair of nodes. Such multiple edges exist because there may more than one distinct physical connection or different network links may only available at different time intervals. By using different levels of information regarding connectivity and/or mobility, routing decisions can be made at individual nodes.

In routing models described above, some level of knowledge regarding node mobility and connectivity is assumed to be available for the routing schemes to work. However, in many cases no such information may be known to the nodes in the network. Under such conditions, different routing approaches are necessary for effective message delivery.

For unicast routing schemes, one or multiple copies can be sent to delivery a message to the destination. Single copy routing schemes use only one copy of the message at a time to transfer the message. Direct transmission is the simplest of single copy routing, where each node will keep its messages until it comes into direct contact with the respective destination nodes. Only one message transfer is made per delivered message, incurring no protocol overhead. Other singlecopy routing schemes include randomized routing, utilitybased routing, etc. [14]. Generally, single copy schemes are more efficient in terms of traffic overhead. However, message delivery ratios are normally lower while delivery delays are higher. One way to improve delivery performance is to use multiple copies of the message taking multiple paths to the destination to increase the likelihood of delivery as well as to decrease delay. Besides each copy can also be divided into multiple chunks [18].

One apparent way to implement a multi-copy scheme is to use flooding. However, due to frequent network partitions, epidemic routing [17] is used as a flooding method in the context of intermittently connected wireless networks. In this schemes, a pair of nodes that come into contact exchanges any missing packets so that they get the same set of messages. Given enough storage space, epidemic routing can be used to reliably disseminate data across the network. By keeping a history of past encounters, nodes can reduce the overhead of epidemic routing [10]. However, due to its large overhead, an uncontrolled flooding scheme such as epidemic routing may not be applicable under circumstances where storage and power supplies are limited. Controlled flooding schemes have been introduced to overcome such problems [7, 13, 15, 17].

There has been a considerable amount of theoretical work in the performance analysis of routing schemes for DTNs [6, 13-15], where message delay is the focus of study. Extensive analysis of the Epidemic routing for performance metrics such as message delivery ratios, message delay, and buffer occupancy are presented in [19] using ordinary differential equations (ODEs). Analysis of multi-copy routing schemes for different performance metrics are studied in [2] using different analytical methods, such recursive method, ODEs, and phase-type distributions. Performance analysis of coreassisted routing for DTNs is given in [1].

The routing approaches described above mostly focus on unicasting. However, there are application scenarios where support for group communication is desirable. While multiple unicasts can be used to perform multicasting, this would be inefficient, especially considering limited network and storage resources available in many DTN applications such as sensor networks. Due to frequent and long partitions, multicasting in DTNs brings new issues to the design of routing algorithms. Besides, as group membership may change while message a being delivered, a set of semantic models are necessary to unambiguously define message delivery semantics in DTNs, as described in [21]. However, the paper mainly discusses the semantic models and simulation scenarios are based on known information regarding node mobility and connectivity. In our work, we mainly concentrate on scenarios where no connectivity information is known in advance and introduce 
various controlled Epidemic routing schemes.

Further, there has been very little work done in the performance analysis of multicast routing schemes. In this study, we define basic performance metrics for multicasting in DTNs and provide performance analysis of fundamental routing schemes for important performance metrics, such as average/full delivery ratio, average/full message delay, and buffer occupancy.

In this paper, we study multicast schemes for DTNs when there is no available knowledge regarding node mobility and connectivity. We use the temporal membership model for multicast delivery semantics described in [21]. We start our evaluations from the Epidemic routing scheme. Then we extend it by introducing TTLs and message expiration times. We also provide performance analysis for basic routing schemes and provide extensive simulation results to demonstrate the effectiveness of different routing schemes and the accuracy of our anlaytical results.

\section{CERM: Controlled EPIDimic Routing FOR Multicasting}

This section describes our controlled Epidemic routing for multicasting, or CERM, protocol. In both wired or dense wireless networks tree-based routing is a common way to implement multicasting. In some cases, broadcast-based or group-based routing can also be used when there is information available regarding node mobility and connectivity [21]. However, such approaches are not readily applicable to DTNs where nodes have to make routing decisions without any knowledge of node connectivity and mobility. Under such network conditions it is hard to form routing trees for multicasting due to network partitions and lack of node connectivity information.

The simplest approach to achieve group delivery might be that the sender will try to deliver its packet to every group member directly. This kind of source based delivery (SBD) is similar to using multiple unicasts to achieve multicasting, and is not really considered a form of multicast. Instead, group-based routing (GBR) can be used, as shown in [21]. In this scheme, members of a group transfer messages for that group only among each other. With an efficient message information exchange mechanism, the protocol overhead of GBR is negligible as group members initiate message transfers only for the messages that they do not have. However, because messages are only transferred within the group lower delivery ratios and higher delays are incurred, specially for small groups. An alternative is to allow different group members to exchange packets.

Epidemic routing (ER) is flooding in the context of DTNs [17], where members of a group exchange packets within their group, as well as members of other groups. It is not hard to see that when no resource constraints are present the Epidemic routing scheme achieves the lowest delay and highest delivery ratio among all possible routing schemes for DTNs. Although Epidemic routing is likely to inapplicable for many application scenarios due to high demand for resources, knowing the lower and upper bounds for message delay and message delivery ratio, respectively, is helpful in evaluating other routing schemes.

Due to aforementioned reasons, we use some form of controlled flooding to implement multicasting in DTNs. We will discuss message transfer policies and procedures in CERM after a general description of performance metrics and mechanisms.

\section{A. Control Mechanisms}

As discussed above, GBR and ER represent two extremes in terms of performance-resource tradeoffs for multicasting applications in DTNs. Now we discuss various controlled Epidemic routing schemes to achieve desired performance goals while saving network resources. Although our basic line of reasoning is similar to that of [7], where controlled flooding for unicasting in DTNs is discussed, we observe that differences exist, especially in the areas pertaining to delivery semantics and intra-group routing policies.

A rich set of policies or rules to control the forwarding behavior can be used in CERM according to specific requirements regarding performance, resource usage, group size, and security, etc.

1) Message Expiration Time: Message expiration time (Time-to-Kill, TTK, or Kill Time [7]) is used to put a timestamp on messages after which they will be dropped. Message expiration times can occur either because of an application requirement or a routing policy. Message expiration time provides a mechanism to control the number of message transfers and buffer occupancy.

2) TTL: The time-to-live field determines the number of hops a message can travel and is used to control the number of message transfers.

3) Probabilistic Routing: This approach is used when nodes attach some probability in their decision to forward a message to a non-group member. It can be used as way to model group members' willingness to forward/receive messages to/from other group members, as well as a way to control the number of message transfers among nodes.

4) Multi-copy Routing: In this approach, the number of copies that are forwarded to non-group members can be controlled similar to Spray and Wait for unicasting [15]. This method is useful for strictly controlling the number of copies that are spread to non-group members.

\section{B. The CERM Protocol}

We assume that nodes are synchronized in time on the order of seconds. The purpose of time synchronization is to allow core nodes to delete messages that are expired. If this is not possible then a standard Time-To-Live mechanism using a countdown timer can be used. We also assume that nodes announce their presence through HELLO messages to their neighbors.

For CERM we assume that all nodes want to multicast messages to other nodes. This is represented as follows: At time $t$ node $i$ produces a message for a destination group with 
id gid. The format of this message is

$$
m s g^{i} \text { (gid, sn, } t, T_{s n}, \text { payload) }
$$

where $s n$ is a sequence number used for duplicate detection, $t$ is the generation time, $T_{s n}$ is the message timeout value, and payload contains the actual data. The timeout value is interpreted as meaning that this message is set to expire at time $t+T_{s n}$. The timeout can be used for memory management and as a means for an application to signify data freshness. All nodes that generate messages are called source nodes.

In CERM, the message transfer procedure is invoked by a node $i$ when a HELLO message is received from $j$, indicating the presence of $j$, along with its node ID and group ID. Intragroup routing policies define the probability of transfer, TTL, number of copies to spread, and TTK if the destination node does not belong to the destination group. When two nodes meet there are four cases to consider.

First, nodes $i$ and $j$ belong to the same group. In this case both nodes examine their queues and determine if they have any messages that are pending for the other node. They then simply exchange the relevant messages, or send to the other node that they have no pending message.

The other three cases deal with the case when nodes $i$ and $j$ are not in the same group. The first considers the case when node $i$ has a message for node $j$ 's group In this case node $i$ delivers the message to node $j$ if $j$ does not have a copy of the message. The second case is when node $i$ has a message for its own group In this case node $i$ may delegate its message to $j$ depending on probabilistic and multi-copy routing policies in effect. The message expiration time and TTL are also set accordingly if the message is transferred. The third considers the case when node $i$ has a message for a third group In this case node $i$ may delegate the message in a way similar to the case above. But different message expiration time, TTL, and probabilities may be used.

We note that each of the intra-group routing schemes discussed above can be used discriminately for different groups. For example, a shorter message expiration time can be set for messages delegated to non-group members so that a message can be spread as quickly as possible at the beginning, while restraining the load of non-group members in the long term. Similar to expiration time, nodes can differentiate between group members and non-group members when setting the TTL and probability of message transfer.

\section{Analysis of Multicasting Routing Schemes}

In this section, we analyze fundamental performance metrics for Epidemic routing including Message Delivery Ratio (MDR) and Delay of Delivered Messages, for both average and full delivery cases. We assume that the inter-arrival time between successive contacts is exponentially distributed. This assumption is supported by the results presented in [6], which shows that nodal inter-meeting times are nearly exponentially distributed when transmission ranges are small compared to the network area size, which is normally the case for opportunistic networks. Although [4] provided evidence that nodal inter-contact times distributed according to power-law based on real-world mobility traces, recent work in [9] presented evidence showing that although the inter-contact time follows power law distribution upto certain time, it shows exponential decay afterwards. Therefore, we believe that our results can be applied to study certain real world application scenarios.

In our analysis, we assume that there are $N+1$ nodes in the system, and use $M$ for the number of nodes in a group. We use $\gamma$ to denote the rate of inter-contact times, and use $\lambda$ to denote the message generation rate at each node. Messages are assumed to have a message expiration time, $T_{x}$, beyond which they will be dropped. Before we discuss the analysis of routing schemes, we first define the performance metrics of interest for multicast routing in DTNs.

\section{A. Performance Metrics}

In unicast, once the destination is found, the packet is delivered and the intermediate or the source node can delete the packet from its buffer. In multicast routing, however, the source or the relays cannot simply delete the packet after it is delivered to one of the members of the target group, as there may be other group members that have not received the packet. At the same time, keep sending copies of a packet to all potential relays also cause redundant packet transfers. One of the objectives of our work is to study the tradeoffs between resource usage and performance in terms of fundamental performance metrics.

In our study we consider the following performance metrics for multicasting:

1) Average message delivery ratio $(A M D R)$

2) Full message delivery ratio $(F M D R)$

3) Average delivery delay

4) Average full delivery delay

5) Buffer occupancy

6) Number of Message Transmissions

Due to differences in unicast and multicast routing models, some performance metrics need to be explained. The AMDR is the ratio of the number of delivered messages to the number of message that should have been delivered. The FMDR is the ratio of the number of messages that are delivered to all group members to the the number message generated for the group. For example, assuming three messages are generated for a group of size 10 and the number of group members that received each message is 9,5 , and 10 , respectively, then $A M D R=(9+5+10) / 30=0.8$, whereas $F M D R=1 / 3$, since only one message is delivered to all of the group members. As we can see, while $A M D R$ measures the overall effectiveness of message delivery to group members, FMDR reflects the effectiveness of a routing scheme to deliver a message to all of the group members. This concept is also extended to message delay.

The number of messages stored in a node buffer is indicated using buffer occupancy. In many of the routing schemes that we consider, buffer occupancy can also be used as an indirect measure of the number of transmissions. This is because the buffer occupancy in the system for a single message is one 
more than the number of transmissions to spread the message when a global message expiration time is used, as each message stored at a node other than the source corresponds to a message transmission.

\section{B. Comparison of CERM Routing Schemes}

We consider two basic routing schemes in our analysis: group-based delivery (GBR) and system-wide Epidemic routing (ER). As discussed in Section III, in GBR nodes exchange messages only among the destination group members, effectively performing Epidemic routing only within that group. With efficient message information exchange among group memebers, the overhead can be considered negligible as group members only exchange messages that need to be delivered. As a result, we assume, in general, that any multicast routing scheme implements some kind of message exchange mechanism that implements GBR on top of any extensions. Therefore, GBR represents a lower bound for the expected message delivery ratio and an upper bound for expected message delivery delay.

System-wide Epidemic routing scheme, on the other hand, represents an extreme case where all the nodes in the system participate in the propagation of a message to its destination group members. Given enough resources, system-wide Epidemic routing achieves the lowest delay and highest message delivery ratio of all possible routing schemes for DTNs, similar to the case of unicast communications, as discussed in [16].

The control mechanisms in CERM are introduced so that a required tradeoff between resource usage and message delivery performance can be achieved, by controlling the spread of a message among non-destination nodes through message expiration times, TTLs, and probabilistic routing, etc.

The group size is also an important factor in considering bounds in the performance of multicast routing schemes. Given that the number of nodes in system is $N+1$, relation $2 \leq M \leq N+1$ holds for group size $M$. The case of $M=2$ represents an extreme case where there are only two nodes in the group. Assuming one of them is the source, GBR reduces to Direct Transmission, a single-copy routing scheme for unicasting in DTNs, where the source holds the message until it meets the destination [14]. Similarly, when $M=2$ system-wide Epidemic routing also reduces to unicast Epidemic routing, for which extensive results are known [6, 19]. This is important as the case $M=2$ represents bounds for certain performance metrics, such as full message delivery ratio, and simplifies the calculation of other metrics, such as average message delivery ratio, as we will discuss later in this section. Under the scenario of $M=N+1$, GBR will be the same as system-wide Epidemic routing. This case represents the upper bound for full message delivery delay and the lower bound for the full message delivery ratio under Epidemic routing scheme for all the possible values of $M$.

Below we study the performance metrics under the cases discussed above when the routing scheme and the group size vary. We do not discuss all the combination, but only focus on certain cases that are useful in understanding the multicasting

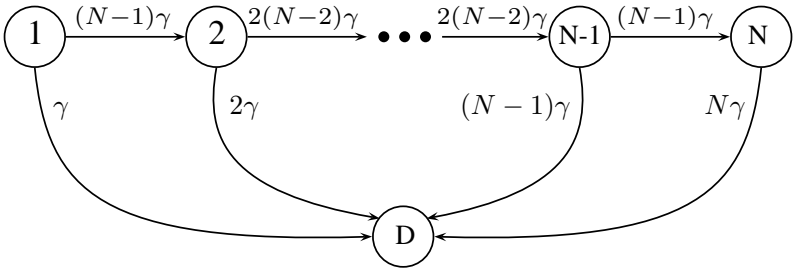

Fig. 1. State Transition Diagram for the Number of Messages in the System (Epidemic routing, unicast)

performance of CERM routing schemes. For simplification we use the notation Case(routing scheme, group size) to denote a specific combination of routing scheme and group size. For example, $\operatorname{Case}(E R, 2)$ denotes the case where system-wide Epidemic routing is used when group size is 2 . In our analysis, we assume that there is a message expiration time, $T_{x}$, and give corresponding result if the assumption $T_{x} \rightarrow \infty$ simplifies an expression.

\section{Average Message Delivery Ratio}

For average message delivery ratio, we mainly focus on Case $(E R, 2)$. In this case system-wide Epidemic routing is used when the group size is 2 , which turns the case into Epidemic unicast. To find the message delivery ratio under a given message expiration time, $T_{x}$, we consider Figure 1, which depicts the state transition diagram for epidemic routing before a copy of the message is delivered a specific node, which is denoted as $D$. To model this we construct a phasetype distribution [11], $\mathrm{PH}\left(\boldsymbol{\alpha}, \boldsymbol{\Theta}_{\mathbf{A}}\right)$, as follows:

$$
\boldsymbol{\Theta}_{A}=\left[\begin{array}{cccccc}
-\gamma_{1} & p_{1} \gamma_{1} & 0 & \ldots & 0 & 0 \\
0 & -\gamma_{2} & p_{2} \gamma_{2} & \ddots & 0 & 0 \\
\vdots & \ddots & \ddots & \ddots & \ddots & \vdots \\
0 & 0 & \ddots & -\gamma_{N-2} & p_{N-2} \gamma_{N-2} & 0 \\
0 & 0 & \ldots & 0 & -\gamma_{N-1} & p_{N-1} \gamma_{N-1} \\
0 & 0 & \cdots & 0 & 0 & -\gamma_{N}
\end{array}\right]
$$

Here $\boldsymbol{\alpha}$ is a $1 \times N$ row matrix, and $\boldsymbol{\Theta}_{A}$ is a $N \times N$ transition matrix, where

$$
\gamma_{i}=\gamma \cdot i(N+1-i), \quad 1 \leqslant i \leqslant N
$$

and

$$
p_{i}=\frac{N-i}{N+1-i}, \quad 1 \leqslant i \leqslant N
$$

Here, $\gamma_{i}$ denotes the rate at which the system leaves state $i$, and $p_{i}$ denotes the probability that the next state is $i+1$. With this, the CDF of message delay, $F_{A}(t)$, can be given as

$$
F_{A}(t)=1-\boldsymbol{\alpha} e^{t \Theta_{A}} \mathbf{1}
$$

where 1 is a column vector of size $N \times 1$, with all elements being one, and $e^{t \boldsymbol{\Theta}_{\mathbf{A}}}$ is the matrix exponential of $t \boldsymbol{\Theta}_{A}$, which 


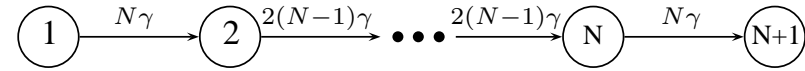

Fig. 2. State Transition Diagram for the Number of Messages in the System (Epidemic routing, multicast, group size: $N+1$ )

is given by:

$$
e^{t \boldsymbol{\Theta}_{\mathbf{A}}}=\sum_{n=0}^{\infty} \frac{t^{n}}{n !} \Theta_{A}^{n}
$$

Using this, we can give the expected average message delivery ratio under message expiration time $T_{x}$ as follows:

$$
E\left[R_{A}\right]=F_{A}\left(T_{x}\right)=1-\boldsymbol{\alpha} e^{T_{x} \boldsymbol{\Theta}_{A}} \mathbf{1}
$$

\section{Average Message Delay}

1) $\operatorname{Case}(E R, 2)$ : Delay characteristics of Epidemic routing scheme has been studied extensively for unicasting [19][6]. When there is a message expiration time, $T_{x}$, the expected delay of delivered messages, $E\left[D_{A}\right]$, can be written using phase-type distribution as follows [2]:

$$
E\left[D_{A}\right]=T_{x}-\frac{1}{F_{A}\left(T_{x}\right)} \int_{0}^{T_{x}} F_{A}(t) d t
$$

Under the simplifying assumption that $T_{x} \rightarrow \infty$, the expected message delay of Epidemic routing, $E\left[D_{A}\right]$, is given by [6] as follows:

$$
E\left[D_{A}\right]=\frac{H_{N}}{\gamma N}, \quad\left(H_{N}=\sum_{i=1}^{N} \frac{1}{i}\right)
$$

where $H_{N}$ is the $N^{\text {th }}$ harmonic number.

2) $\operatorname{Case}(E R, M)$ : In this case, we consider the cases where $M>2$. Under the assumption that node movements are i.i.d, the average message delay under multicasting scheme is the same as $E\left[D_{A}\right]$ given in (4). This is because the expected delay for each node is the same and is equal to $E\left[D_{A}\right]$. This also means that the expected average message delay is independent of the size of the multicast group.

\section{E. Full Message Delivery Ratio}

1) Case $(E R, 2)$ : Under this scenario, the full message delivery ratio will be the same as the average message delivery ratio for $\operatorname{Case}(E R, 2)$, as given in Equation (2), since there is only one destination node. This case represents the upper bound for full message delivery ratio when varying the group size $M$, as any increase in the group size will lower the likelyhood of full delivery.

2) $\operatorname{Case}(E R, N+1)$ : This case represents the lower bound for the full message delivery ratio for varying group size $M$, as all the nodes in the system need to receive the message under this scenario.

To analyze this case for the full message delivery ratio, we consider the graph shown in Figure 2. With this state transition diagram we construct phase-type distribution $\operatorname{PH}\left(\boldsymbol{\alpha}, \boldsymbol{\Theta}_{F}\right)$, where $\boldsymbol{\Theta}_{F}$ is constructed the same way as $\boldsymbol{\Theta}_{A}$, except that $p_{i}=1$ for all $1 \leqslant i \leqslant N$. Following Equation (1), we give the $\mathrm{CDF}$ for full message delay, $F_{F}(t)$, as follows:

$$
F_{F}(t)=1-\boldsymbol{\alpha} e^{t \Theta_{F}} \mathbf{1}
$$

Consequently, the expected full message delivery ratio under time constraint $T_{x}$ is given as

$$
E\left[R_{F}\right]=F_{F}\left(T_{x}\right)=1-\boldsymbol{\alpha} e^{T_{x} \boldsymbol{\Theta}_{F}} \mathbf{1}
$$

3) $\operatorname{Case}(G B R, M)$ : For any group of size $M$, the expected full message delivery ratio under the GBR scheme can also be obtained from Equation (6) by replacing $N$ with $M$, as GBR is equivalent of performing Epidemic routing within the group.

\section{F. Full Message Delivery Delay}

1) $\operatorname{Case}(E R, 2)$ : Under this scenario, the full message delivery delay will be the same as the average message delivery delay for $\operatorname{Case}(E R, 2)$, as discussed above, and Equation (3) can be used to calculate full message delay. This case represents the lower bound for full message delivery delay when varying the group size $M$, as any increase in the group size will increase the time that it takes to delivery a message to all of the group members. Under the assumption that $T_{x} \rightarrow \infty$, Equation (4) can be used to obtain the full delivery delay.

2) $\operatorname{Case}(E R, N+1)$ : This case represents the upper bound for the expected full message delivery delay, as the message needs to be delivered to all of the nodes in the system for full delivery. Using the phase-type distribution that we constructed for full message delivery ratio, we can give the expected full message delay, $E\left[D_{F}\right]$, as follows

$$
E\left[D_{F}\right]=T_{x}-\frac{1}{F_{F}\left(T_{x}\right)} \int_{0}^{T_{x}} F_{F}(t) d t
$$

Under the condition that $T_{x} \rightarrow \infty$, the expression above can be given as follows using the state transition diagram in Figure 2:

$$
\begin{aligned}
E\left[D_{F}\right] & =\sum_{i=1}^{N} \frac{1}{i(N+1-i) \gamma} \\
& =\frac{1}{\gamma(N+1)} \sum_{i=1}^{N}\left(\frac{1}{i}+\frac{1}{N+1-i}\right) \\
& =\frac{2 H_{N}}{\gamma(N+1)}
\end{aligned}
$$

where $H_{N}$ is the $N^{\text {th }}$ harmonic number.

Since $N+1$ is the maximum size for any group, $E\left[D_{F}\right]$ obtained represents the upper bound for the expected full message delivery delay for a group for any message expiration time. The lower bound is given in the $\operatorname{Case}(E R, 2)$ above, in which the full message delay is the same as the average message delay, given in Equation (4). Therefore, we can see that the full message delay is lower bounded by $E\left[D_{A}\right]$, and is upper bounded by $E\left[D_{F}\right]$. We also note that $E\left[D_{F}\right] \approx$ $2 E\left[D_{A}\right]$ for large $N$. That is, the upper bound of expected 
full message delivery delay is approximately twice as large as the average message delay when $N$ is large.

3) $\operatorname{Case}(G B R, M)$ : For any group of size $M$, the expected full message delay under the GBR scheme can also be obtained from Equations (7) and (8) by replacing $N+1$ with $M$, as GBR is equivalent of performing Epidemic routing within the group.

\section{G. Buffer Occupancy}

Deriving the buffer occupancy by directly using phase-type distribution seems difficult. For this reason, we use a recursion method to calculate the expected buffer occupancy at nodes. We assume that the number of nodes participating in message exchange is $S+1$. It is not hard to see that $S$ is equal to $N$ under system-wide Epidemic routing, and is equal to $M-1$ when GBR is used. In this method, we use state $i$ to denote the number of nodes have a copy of a specific message, and for each state $i$ we consider the rate at which the system leaves state $i$ and the probabilities of the system moving to the next state $i+1$.

If we consider $\operatorname{Case}(E R, 2)$ and assume that an intermediate node knows to delete message just after it has delivered the copy that it has, this scheme represents a lower bound on the expected buffer occupancy.

When the system is at state $S$ with expiration time $t_{x}$, the number of message in the system is given as $S(\lambda / \gamma)\left(1-e^{\gamma t_{x}}\right)$ [2]. At state $i$, where $i<S$, the source gives out a copy, either to the destination, or to another node that has not received a copy of the message, at a rate of $i *(S-i+1) \gamma$. The probability of delivering it to the destination is $1 /(S-i+1)$, and the probability of delivering to another relay is $(S-i) /(S-i+1)$. Based on the analysis, the expected buffer occupancy, $E[Q]$, at each node can be expressed as follows, where $E[Q] \equiv$ $E Q_{x}\left(1, T_{x}, S\right)$ :

$$
\begin{gathered}
E Q_{x}\left(i, t_{x}, s\right)=i \cdot \lambda \cdot E D_{i, s}+ \\
\frac{1}{s-i+1} E Q_{x}\left(i-1, t_{x}-E D_{i, s}, s-1\right)+ \\
\frac{s-i}{s-i+1} E Q_{x}\left(i+1, t_{x}-E D_{i}, s\right), \quad i \in[1, S-1] \\
E Q_{x}\left(i, t_{x}, s\right)=s * \frac{\lambda}{\gamma} \cdot F\left(\gamma, t_{x}\right), \quad i=S
\end{gathered}
$$

Here, $F\left(\gamma, t_{x}\right)=1-e^{-\gamma t_{x}}$, and $E D_{i, s}=\Phi\left((s-i+1) \gamma, t_{x}\right)$, where function $\Phi\left(\gamma, t_{x}\right)$ gives the expected delay of a message with expiration time $t_{x}$ when the inter-contact rate of the destination is $\gamma$, and is defined as [2]:

$$
\Phi\left(\gamma, t_{x}\right)=\frac{1}{\gamma}-\frac{e^{-\gamma t_{x}}}{1-e^{-\gamma t_{x}}} t_{x} .
$$

It can be shown that the value of $\Phi\left(\gamma, t_{x}\right)$ is upper bounded by $\min \left\{t_{x}, 1 / \gamma\right\}$, and approaches $1 / \gamma$ when $t_{x} \rightarrow \infty$.

In the calculation of buffer occupancy above, we equated the buffer occupancy at each node with the total buffer occupancy of the system caused by the message generation at a single node, which is given by the set of equations in (9). The reason is as follows. Assuming the total expected buffer occupancy

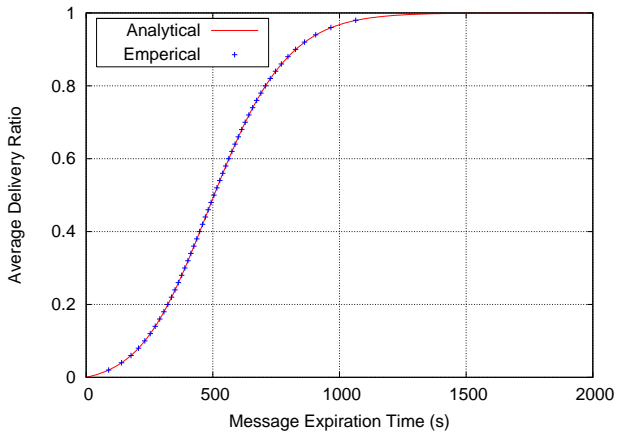

(a) Average MDR

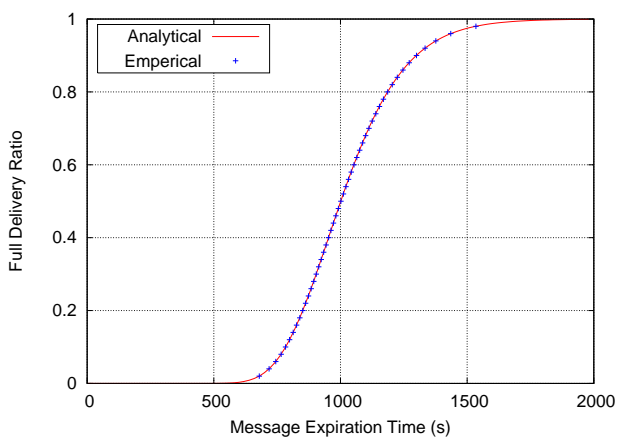

(b) Full MDR

Fig. 3. Average and Full Message Delivery Ratios under Varying Message Expiration Times (exponential inter-contact times in custom simulator)

in the system (including the source) caused by the message generation at a single node is $Q$, the expected total buffer occupancy of all the nodes of the system is given by $S \times Q$, as there are $S$ nodes in the system. Since each of the $S$ nodes in the system is equally likely to share the total buffer occupancy of the system, the expected buffer occupancy at each node is again given by $Q$.

\section{EXPERIMENTAL RESUlts}

In this section, we present our experimental results. The goal our experiments is to check the correctness of our analytical results, as well as to examine performance tradeoffs when varying routing parameters.

\section{A. Experimental Settings}

Most of our experiments use the $n s-2$ network simulator extended with our own code. We also use our custom simulator to experiment with exponential inter-contact times.

The default settings for $n s$ - 2 simulations are as follows. Each simulation run has 60 nodes moving according to Random Waypoint model in a $6000 \mathrm{~m} \times 6000 \mathrm{~m}$ square area. By default, nodes have a radio range of $250 \mathrm{~m}$. Minimum and maximum speeds, $v_{\min }$ and $v_{\max }$, are $3 \mathrm{~m} / \mathrm{s}$ and $10 \mathrm{~m} / \mathrm{s}$, respectively. The HELLO interval is set to 3 seconds. By default there are 3 groups in the system, and we change the number of groups in the range of 1-30 in our experiments, corresponding to group size in the range of 2-60 nodes. 


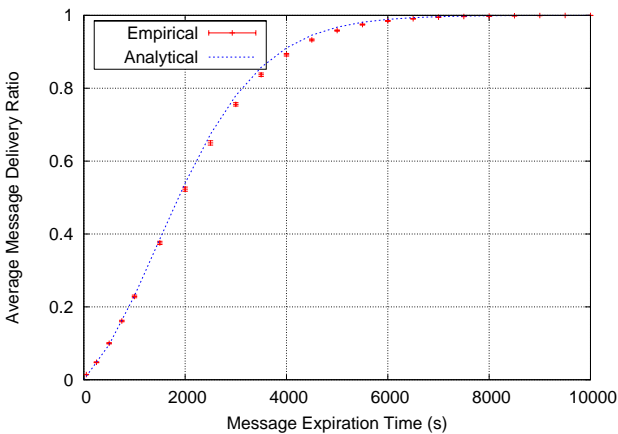

(a) Average MDR

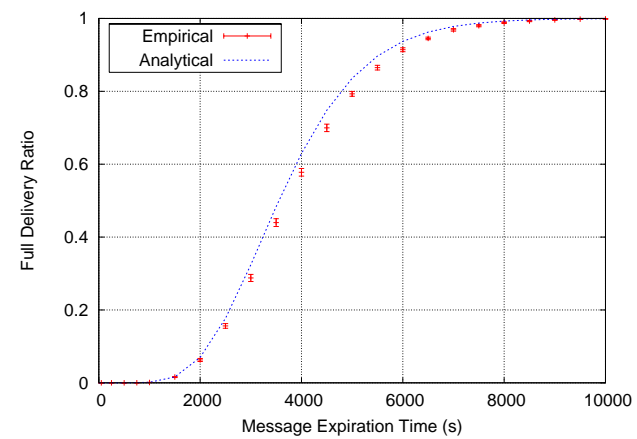

(b) Full MDR

Fig. 4. Average and Full Message Delivery Ratios under Varying Message Expiration Times (Random Waypoing model in $n s-2$, group size: 10 )

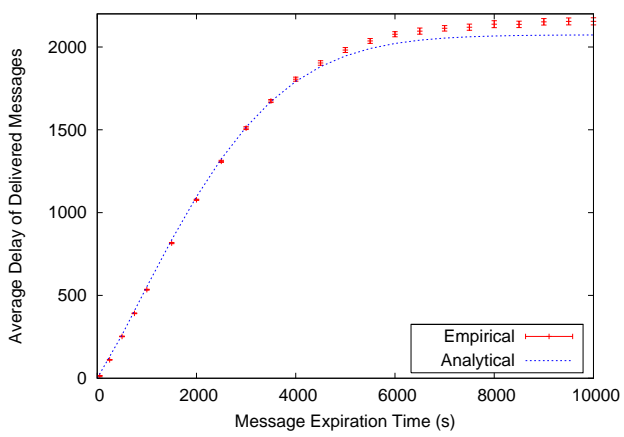

(a) Average Message Delay

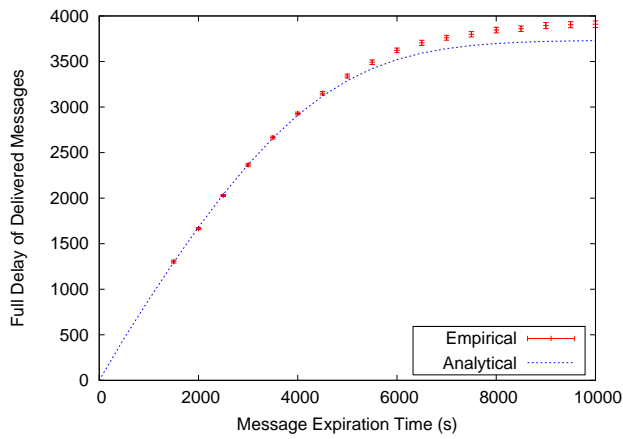

(b) Full Message Delay

Fig. 5. Average and Full Message Delay of Delivered Messages under Varying Message Expiration Times (Random Waypoing model in $n s$-2, group size: 10$)$

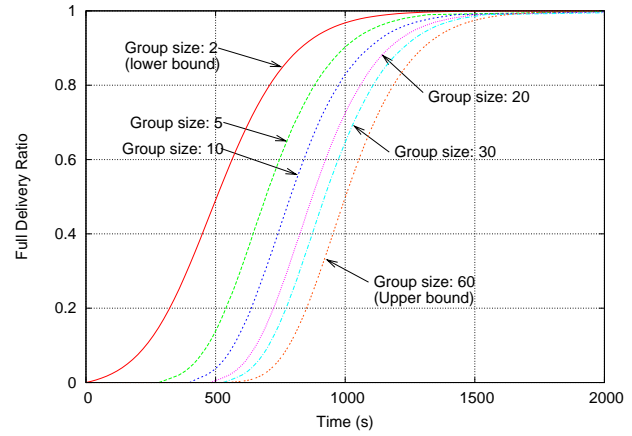

Fig. 6. Full MDR under Varying Group Size

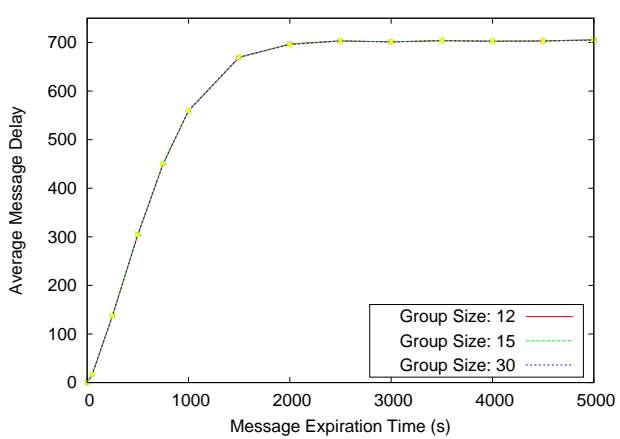

Fig. 7. Average Message Delay in Epidemic Routing Scheme with Varying Group Sizes: $M=12,15,30$.

\section{B. Experimental Results}

Figure 3(a) shows the empirical and analytical results for average message delivery ratio. The empirical results are obtained from our custom simulator using poisson arrivals for nodal inter-contact times. Analytical results are obtained from Equation (1). We can see the experimental and analytical results agree closely.

Empirical results for average and full message delivery ratios obtained from $n s$ - 2 when nodes move according to the Random Waypoint model are shown in Figure 4. We can see that the analytical results agree with the empirical results.

Experimental results obtained in $n s-2$ for average and full message delivery delays are shown in Figure 5. Analytical results are calculated using Equations (3) and (7), respectively.

Figure 6 shows the variations in FMDR when we change the group size. The smallest group size is 2 , and the largest group size is 60 , which is the total number of nodes in the system in our experiments. We can see that the FMDR values when the group size is varied are within the lower and upper bounds. We also notice that the upper bound for the expected full message delivery ratio, when $M=60$, is about twice as large as the lower bound for the expected full message delivery ratio, when $M=2$, as discussed in the previous section.

Figure 7 shows the average message delay under systemwide epidemic routing scheme when the group size takes different values: $M=12,15,30$. As we can see, there is no significant difference in the average delay when group size varies, consistent with our analysis in Section IV. 


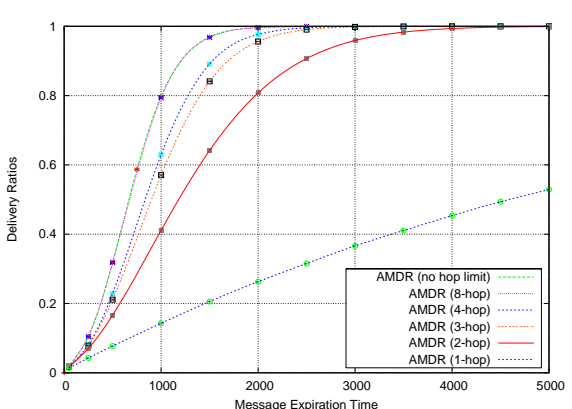

(a) Average MDR

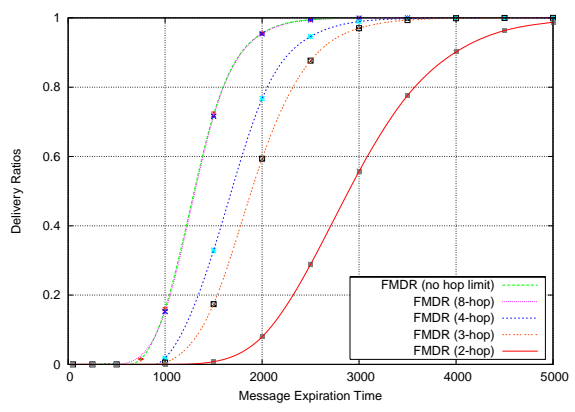

(b) Full MDR

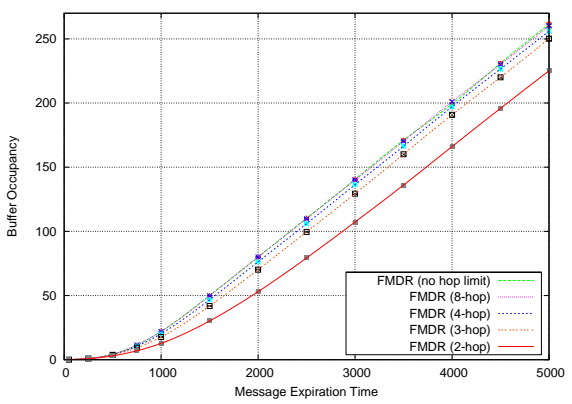

(c) Buffer Occupancy

Fig. 8. Message Delay under Varying TTL

Figure 8 shows the variations in average message delivery ratios, full message delivery ratios, and buffer occupancy when the TTL for non-group members are changed from 1 to 8. As can bee seen, different levels of controls can be achieved through the use of inter-group routing policies. In this scenario, when a destination group member that has a copy of a specific message meets a non-destination group member, the destination group member sets the TTL of the message to a value specified by the policy. This is to control the spread of message among non-group members, and reduce the number of transmissions. As we can see, changing the TTL in the routing policy has different effects on average and full message delivery ratios, as well as on the buffer occupancy. Under specific scenarios, the TTL can be varied to achieve a desired performance requirement. For example, given the requirement that average message delivery ratio should at least be $95 \%$ when the message expiration time is $3000 \mathrm{~s}$, we may choose $T T L=2$, instead of 3,4 or 8 , to decrease the buffer occupancy and the number of transmissions. However, if it is required that the full message delivery ratio should at least be 95\% under the same message expiration time, $T T L=3$ will be sufficient. Other mechanisms in CERM, such as message expiration times, routing probabilities, and number of copies can be used to control inter-group routing policies.

\section{CONCLUSiOns AND Future Work}

In this paper, we studied multicasting in delay tolerant networks (DTNs). We proposed CERM: Controled Epidemic Routing for Multicasting in DTNs. CERM is a collection of schemes to achieve efficient message delivery for multicasting through the use of controlled Epidemic routing. The basic mechanisms used in CERM are message expiration times, TTLs, forwarding probabilities, and the number of copies to spread. These mechanisms help define routing policies between destination group members and non-destination group members to achieve a balance in the tradeoffs between resource usage and message delivery performance. We also analyzed two different routing schemes, group based deliver (GBR) and system-wide epidemic routing, under basic routing scenarios to derive some upper and lower bounds for important performance metrics such as message delivery ratios, message delays, and buffer occupancy. Through extensive experiments, we showed that controlling the performance of multicasting routing in DTNs is possible through the use of CERM mechanisms, and that our analytical results are accurate.

\section{REFERENCES}

[1] Muhammad Abdulla and Robert Simon. Analysis of core-assisted routing in opportunistic networks. In MASCOTS'07, Istanbul, Turkey, Oct. 2007.

[2] Muhammad Abdulla and Robert Simon. Analytical techniques for performance analysis of multi-copy routing schemes in delay tolerant networks. Technical Report CS-200729, George Mason University, 2007.

[3] Scott Burleigh and Kevin Fall. Delay tolerant networking: An approach for interplanetary internet. IEEE Communications Magazine, June 2003.

[4] A. Chaintreau, P. Hui, J. Crowcroft, C. Diot, R. Gass, and J. Scott. Impact of human mobility on the design of opportunistic forwarding algorithms. In INFOCOM, pages 1-13, April 2006.

[5] Kevin Fall. A delay-tolerant network architecture for challenged internets. Karlsruhe, Germany, August 2003. ACM SIGCOMM'03.

[6] R. Groenevelt, P. Nain, and G. Koole. The message delay in mobile ad hoc networks. In Performance, 2005.

[7] K. A. Harras, K. C. Almeroth, and E. M. Belding-Royer. Delay tolerant mobile networks: Controlled flooding in sparse mobile networks. In Proceedings of Networking, Waterloo, Ontario, Canada, May 2005.

[8] Sushant Jain, Kevin Fall, and Rabin Patra. Routing in a delay tolerant network. ACM SIGCOMM'04, August 2004.

[9] T. Karagiannis, J.-Y. Le Boudec, and M. Vojnovic. Power law and exponential decay of inter contact times between mobile devices. Technical Report MSR-TR-2007, Microsoft Research, March 2007.

[10] A. Lindgren, A. Doria, and O. Scheln. Probabilistic routing in intermittently connected networks, 2003.

[11] M. F. Neuts. Matrix-Geometric Solutions in Stochastic Models. Dover Publications; Rev. Ed., 1995.

[12] R. Shah, S. Roy, S. Jain, and W. Brunette. Data mules: Modeling a three-tier architecture for sparse sensor networks. IEEE SNPA, 2003.

[13] T. Small and Z. J. Haas. Resource and performance tradeoffs in delaytolerant wireless networks. In WDTN '05, pages 260-267, 2005.

[14] T. Spyropoulos, K. Psounis, and C. Raghavendra. Single-copy routing in intermittently connected mobile networks. In Sensor and Ad Hoc Communications and Networks, pages 235-244. IEEE, Oct 2004.

[15] T. Spyropoulos, K. Psounis, and C. Raghavendra. Spray and wait: An efficient routing scheme for intermittently connected mobile networks. Philadelphia, PA, USA, August 2005. ACM SIGCOMM'05 Workshops. 
[16] T. Spyropoulos, K. Psounis, and C. Raghavendra. Performance analysis of mobility-assisted routing. pages 49-60. ACM Mobihoc 2006, 2006.

[17] A. Vahdat and D. Becker. Epidemic routing for partially connected ad hoc networks. Technical Report CS-200006, Duke University, 2000.

[18] Yong Wang, Sushant Jain, Margaret Martonosi, and Kevin Fall. Erasurecoding based routing for opportunistic networks. Philadelphia, PA, USA August 2005. ACM SIGCOMM'05 Workshops.

[19] X. Zhang, G. Neglia, J. Kurose, and D. Towsley. Performance modeling of epidemic routing. Technical Report CMPSCI 2005-44, UMass, 2005.

[20] W. Zhao, M. Ammar, and E. Zegura. A message ferrying approach for data delivery in sparse mobile ad hoc networks. MobiHoc, May 2004

[21] Wenrui Zhao, Mostafa Ammar, and Ellen Zegura. Multicasting in delay tolerant networks: Semantic models and routing algorithms. In SIGCOMM'05 Workshops, pages 268-275. ACM, August 2005. 